\title{
ON CONVERGENCE IN ELLIPTIC SHAPE OPTIMIZATION
}

\author{
KARSTEN EPPLER ${ }^{\dagger}$, HELMUT HARBRECHT ${ }^{\ddagger}$, AND REINHOLD SCHNEIDER $§$
}

\begin{abstract}
This paper is aimed at analyzing the existence and convergence of approximate solutions in shape optimization. Two questions arise when one applies a Ritz-Galerkin discretization to solve the necessary condition: does there exists an approximate solution and how good does it approximate the solution of the original infinite dimensional problem? We motivate a general setting by some illustrative examples, taking into account the so-called two norm discrepancy. Provided that the infinite dimensional shape problem admits a stable second order optimizer, we are able to prove the existence of approximate solutions and compute the rate of convergence. Finally, we verify the predicted rate of convergence by numerical results.
\end{abstract}

Key Words: shape optimization, shape calculus, existence and convergence of approximate solutions, optimality conditions.

AMS Subject Classification: 49Q10, 49K20, 49M15, 65K10.

1. Introduction. Shape optimization is quite indispensable for designing and constructing industrial components. Many problems that arise in application, particularly in structural mechanics and in the optimal control of distributed parameter systems, can be formulated as the minimization of functionals defined over a class of admissible domains. Therefore, such problems have been intensively studied in the literature throughout the last 25-30 years (see [26, 28, 33, 36], and the references therein). However, only few attempts have been made to investigate the convergence of approximate solutions to the solution of the original shape optimization problem in more detail.

In $[14,15,16,18,19,20,21]$, we considered the numerical solution of several elliptic shape optimization problems. There, a simple boundary variational approach was used in combination with boundary integral representations of the shape gradient and the shape Hessian. It allows the embedding of related shape problem into a Banach space by identifying the domain with the parametrization of its boundary. Then, a Ritz-Galerkin-like discretization of the shape optimization problem was introduced. All ingredients of the shape gradient and shape Hessian, that arise from the state equation, were computed with sufficiently high accuracy by a fast wavelet boundary element method. This concept decouples the discretization of the shape from the discretization of the state equation. As an important consequence, this enables us to consider the convergence of the semidiscretized shape problems, that is, only the discretization with respect to the shape has to be considered. Whereas the numerical results confirm a good approximation behaviour to solutions of the original problems, the question of convergence analysis remains still open.

Identifying the domain with its parametrization with respect to a fixed reference manifold, a shape calculus which is based on fixed variational fields leads to a second

${ }^{*}$ This research has been carried out when the second author stayed at the Department of Mathematics, University Utrecht, Netherlands, supportet by the EU-IHP project Nonlinear Approximation and Adaptivity: Breaking Complexity in Numerical Modelling and Data Representation

${ }^{\dagger}$ Weierstraß Institut für Angewandte Analysis und Stochastik, Mohrenstr. 39, 10117 Berlin, Germany, e-mail:eppler@math.wias-berlin.de

${ }^{\ddagger}$ Institut für Informatik und Praktische Mathematik, Christian-Albrechts-Universität zu Kiel, Olshausenstr. 40, 24098 Kiel, Germany, e-mail: hh@numerik.uni-kiel.de

§Institut für Informatik und Praktische Mathematik, Christian-Albrechts-Universität zu Kiel, Olshausenstr. 40, 24098 Kiel, Germany, e-mail: rs@numerik.uni-kiel.de 
order Frechét calculus in a Banach space, cf. [11, 12, 13]. Given now a stationary domain of the optimization problem under consideration, optimality can be guaranteed often by some coercivity of the second order Frechét derivative. However, it turns out that coercivity can be realized only in a weaker space, usually in the context of elliptic shape optimization a Sobolev space $H^{s}$. This lack of regularity is known from other pde-constrained optimal control problems as the so-called two norm discrepancy, cf. $[3,4,23,24]$. The two norm discrepancy in shape optimization has been observed first in $[6,7,8,11,13]$.

Section 2 is dedicated to overview on second order shape calculus. Additionally, we give some examples to illustrate particular cases of the two norm discrepancy. First, we consider shape functionals based on a simple domain or boundary integral. Then, we treat pde-constraint shape optimization problems by means of elliptic free boundary problems. Finally, for sake of completeness, we shall also have a look to shape optimization problems with additional functional constraints.

Motivated by these examples, we present in Section 3 an abstract setting for the investigation of the second order sufficient optimality condition to verify stable minimizers. Then, we introduce suitable trial spaces to discretize the shape optimization problem by means of a Ritz-Galerkin method for solving the necessary condition. The Ritz-Galerkin method solves a finite dimensional optimization problem, that arises from restricting the class of admissible domains to those domains given by the trial space. We show that there exist approximate solutions provided that the level of discretization is sufficiently large and prove convergence of the approximate solutions $\Omega_{N}$ to $\Omega^{\star}$, the optimal solution of the original infinite dimensional shape problem. The approximate solution behaves like the best approximation in the trial space to $\Omega^{\star}$, with respect to the natural space of coercivity of the shape Hessian. Therefore, the computation of the rate of convergence is along the lines of a conventional approximation theory.

In Section 4, we present two numerical examples that confirm our analysis. The first one is a simple shape problem based on a domain integral minimization, which is mainly incorporated for illustration. The second is a more advanced pde-constrained shape optimization problem, with several additional functional constraints. Both examples are chosen such that the optimal domain is known a priori. We observe rates of convergence which verify to the present theory.

\section{Motivation and Background.}

2.1. Shape Calculus. Shape optimization is concerned with the minimization of the shape functional

$$
J(\Omega)=\int_{\Omega} j(u, \nabla u, \mathbf{x}) d \mathbf{x} \rightarrow \min , \quad \Omega \in \Upsilon,
$$

where $\Upsilon$ is a suitable class of admissible domains $\Omega \in \mathbb{R}^{n}$. The so-called state $u$ satisfies an abstract free boundary problem

$$
\begin{aligned}
& \mathcal{L} u=0, \quad \text { in } \Omega, \\
& \mathcal{B} u=0, \quad \text { on } \Gamma,
\end{aligned}
$$

where $\mathcal{L}$ corresponds to a well posed elliptic boundary value problem in the domain $\Omega$ and $\mathcal{B}$ operates on the functions supported at the free boundary $\Gamma \subset \partial \Omega$. For sake of simplicity, we restrict ourselves to finding solutions with known topology and assume all involved functions and data sufficiently smooth. 
Generally, the problem (2.1) is highly implicit with respect to the shape of the domain and has to be solved iteratively. The canonical way to solve the minimization problem is to determine its stationary points. Then, via the second order optimality condition regular minimizers of second order are verified. To this end, we will briefly survey on shape calculus. In particular, we refer the reader for example to Murat and Simon [30, 35], Pironneau [33], Sokolowski and Zolesio [36], Delfour and Zolesio [10], and the references therein. Herein, two basic concepts are considered, namely, the perturbation of identity (Murat and Simon) and the speed method (Sokolowski and Zolesio).

For example, the perturbation of identity exploits a smooth perturbation field $\mathbf{U}: \Omega \rightarrow \mathbb{R}^{n}$ and defines the standard domain perturbation as

$$
\Omega_{\varepsilon}[\mathbf{U}]:=\{(\mathbf{I}+\varepsilon \mathbf{U})(\mathbf{x}): \mathbf{x} \in \Omega\} .
$$

Then, the directional derivative of $J(\Omega)$ is computed as

$$
\nabla J(\Omega)[\mathbf{U}]:=\lim _{\varepsilon \rightarrow 0} \frac{J\left(\Omega_{\varepsilon}[\mathbf{U}]\right)-J(\Omega)}{\varepsilon} .
$$

It is known since Hadamard [25] that $\nabla J(\Omega)[\mathbf{U}]$ is a distribution living only on the free boundary of the domain $\Omega$ provided that $J(\Omega)$ is shape differentiable, see also [9].

The latter observation leads to the idea to consider simply boundary variations for the update in the optimization algorithms. Hence, it is quite convenient to apply directly boundary variations for the computation of the boundary integral representations of the shape gradient and Hessian. To this end, we introduce an $n$-dimensional reference manifold $\Gamma_{0}$ and consider a fixed boundary perturbation field, for example in direction of the outer normal $\mathbf{n}_{0}$. We suppose that the free boundary of each domain $\Omega \in \Upsilon$ can be parameterized via a sufficiently smooth function $r$ in terms of

$$
\gamma: \Gamma_{0} \rightarrow \Gamma, \quad \gamma(\mathbf{x})=\mathbf{x}+r(\mathbf{x}) \mathbf{n}_{0}(\mathbf{x}) .
$$

That is, we can identify a domain with the scalar function $r$. Defining the standard variation

$$
\gamma_{\varepsilon}: \Gamma_{0} \rightarrow \Gamma_{\varepsilon}, \quad \gamma_{\varepsilon}(\mathbf{x}):=\gamma(\mathbf{x})+\varepsilon d r(\mathbf{x}) \mathbf{n}_{0}(\mathbf{x}),
$$

where $d r$ is again a sufficiently smooth scalar function, we obtain the perturbed domain $\Omega_{\varepsilon}$. Consequently, both, the shape and its increment, can be seen as elements of a Banach space $X$. We will specify the notion "sufficiently smooth" in the next subsections.

2.2. Optimization of Domain or Boundary Integrals. First we introduce some notation. For a given domain $D \in \mathbb{R}^{n}$ the space $C^{2}(\bar{D})$ consists of all two times continuously differentiable functions $f: \bar{D} \rightarrow \mathbb{R}^{m}$. A function $f \in C^{2}(\bar{D})$ belongs to $C^{2, \alpha}(\bar{D})$, if the (spatial) Hessian $\nabla^{2} f$ is Hölder continuous with coefficient $0<\alpha \leq 1$. A domain $D \in \mathbb{R}^{n}$ is of class $C^{2, \alpha}$ if for each $\mathbf{x} \in \partial D$ a neighborhood $U(\mathbf{x}) \subseteq \partial \Omega$ and a diffeomorphism $\left.\gamma:[0,1]^{n-1} \rightarrow \overline{U(\mathbf{x}}\right)$ exists such that $\gamma \in C^{2, \alpha}\left([0,1]^{n-1}\right)$, see $[38]$ for example.

For sake of clearness, we present here two elementary shape problems, since both, the shape calculus and the analysis, become much more evident in comparison to the more advanced shape optimization problems presented in the subsequent subsections. 
To this end, let $n=2, \Omega \in C^{1}$, and consider the following shape optimization problem of domain integral type

$$
J(\Omega)=\int_{\Omega} h(\mathbf{x}) d \mathbf{x} \rightarrow \min ,
$$

where $h \in C^{1}\left(\mathbb{R}^{2}\right)$ are given data. We choose the class of admissible domains as the set of all domains which are starshaped with respect to the origin. Then, we can choose $\Gamma_{0}$ as the unit circle. Equivalently, we can parameterize $\Gamma=\partial \Omega$ via polar coordinates

$$
\Gamma:=\left\{\gamma(\phi)=r(\phi)\left[\begin{array}{c}
\cos \phi \\
\sin \phi
\end{array}\right]: \phi \in[0,2 \pi]\right\},
$$

where $r \in C_{\mathrm{per}}^{1}([0,2 \pi])$ is a positive function. Here and in the sequel, the space $C_{\mathrm{per}}^{k, \alpha}$ is defined as

$$
C_{\mathrm{per}}^{k, \alpha}([0,2 \pi])=\left\{f \in C^{k, \alpha}([0,2 \pi]): f^{(i)}(0)=f^{(i)}(2 \pi) \text { for all } i=0, \ldots, k\right\},
$$

and likewise $C_{\mathrm{per}}^{k}([0,2 \pi])$. Let us further remark that the tangent and the outer normal at $\Gamma$ are computed by

$$
\mathbf{t}=\frac{r^{\prime}\left[\begin{array}{c}
\cos \phi \\
\sin \phi
\end{array}\right]+r\left[\begin{array}{r}
-\sin \phi \\
\cos \phi
\end{array}\right]}{\sqrt{r^{2}+r^{\prime 2}}}, \quad \mathbf{n}=\frac{r^{\prime}\left[\begin{array}{r}
\sin \phi \\
-\cos \phi
\end{array}\right]+r\left[\begin{array}{c}
\cos \phi \\
\sin \phi
\end{array}\right]}{\sqrt{r^{2}+r^{\prime 2}}} .
$$

We consider $d r \in C_{\mathrm{per}}^{1}([0,2 \pi])$ as standard variation for perturbed domains $\Omega_{\varepsilon}$ and boundaries $\Gamma_{\varepsilon}$, respectively, defined by $r_{\varepsilon}(\phi)=r(\phi)+\varepsilon d r(\phi)$, where $\boldsymbol{\gamma}_{\varepsilon}(\phi)=r_{\varepsilon}(\phi) \mathbf{n}_{0}(\phi)$ is always a Jordan curve. Herein, $\mathbf{n}_{0}(\phi)=[\cos \phi, \sin \phi]^{T}$ denotes the outer normal vector to the reference manifold $\Gamma_{0}$.

Lemma 2.1 ([12]). The shape functional from (2.3) is twice Frechét differentiable with respect to $C_{\mathrm{per}}^{1}([0,2 \pi])$, where the shape gradient and Hessian read as

$$
\begin{gathered}
\nabla J(\Omega)[d r]=\int_{0}^{2 \pi} r(\phi) d r(\phi) h(r(\phi), \phi) d \phi \\
\nabla^{2} J(\Omega)\left[d r_{1}, d r_{2}\right]=\int_{0}^{2 \pi} d r_{1}(\phi) d r_{2}(\phi)\left\{h(r(\phi), \phi)+r(\phi) \frac{\partial h}{\partial \mathbf{n}_{0}}(r(\phi), \phi)\right\} d \phi .
\end{gathered}
$$

Consider now a stationary domain $\Omega^{\star}$, which means $\nabla J\left(\Omega^{\star}\right)[d r]=0$ for all $d r \in$ $C^{1}([0,2 \pi])$. Of course, the latter equation implies that $\left.h\right|_{\Gamma^{\star}} \equiv 0$. Hence, as one readily verifies, it holds

$$
\nabla^{2} J\left(\Omega^{\star}\right)\left[d r_{1}, d r_{2}\right]=\int_{0}^{2 \pi} d r_{1}(\phi) d r_{2}(\phi)\left\{\frac{r^{\star 2}(\phi)}{\sqrt{r^{\star 2}(\phi)+r^{\star \prime}(\phi)}} \frac{\partial h}{\partial \mathbf{n}}\left(r^{\star}(\phi), \phi\right)\right\} d \phi
$$

Optimality can be guaranteed often by some coercivity of the second order Frechét derivative. However, it is impossible to realize coercivity with respect to $C_{\mathrm{per}}^{1}([0,2 \pi])$, only an estimate

$$
\nabla^{2} J\left(\Omega^{\star}\right)[d r, d r] \geq c_{E}\|d r\|_{L^{2}([0,2 \pi])}^{2}
$$

for some $c_{E}>0$ can be expected. Note that we have such an estimate if $\left.(\partial h / \partial \mathbf{n})\right|_{\Gamma^{*}} \geq$ $c_{E}>0$. This lack of regularity is known from other control problems as the so-called 


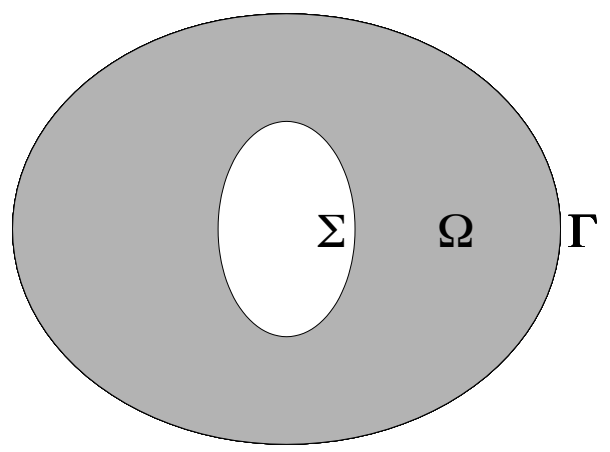

FIG. 2.1. The domain $\Omega$ and its boundaries $\Gamma$ and $\Sigma$.

two norm discrepancy. Nevertheless, the bilinear form imposed by the shape Hessian $\nabla^{2} J(\Omega)$ is obviously also continuous on $L^{2}([0,2 \pi]) \times L^{2}([0,2 \pi])$, that is

$$
\left|\nabla^{2} J(\Omega)\left[d r_{1}, d r_{2}\right]\right| \leq c_{S}(\Omega)\left\|d r_{1}\right\|_{L^{2}([0,2 \pi])}\left\|d r_{2}\right\|_{L^{2}([0,2 \pi])}
$$

for all $d r_{1}, d r_{2} \in L^{2}([0,2 \pi])$. Notice that it is generally impossible to extend the domain of definition $C^{1}([0,2 \pi])$ to $L^{2}([0,2 \pi])$. In other words, $J$ is only densely defined with respect to $L^{2}([0,2 \pi])$.

Also in the case of shape optimization problem of boundary integral type

$$
J(\Omega)=\int_{\Gamma} g(\mathbf{x}) d \sigma \rightarrow \min ,
$$

where $g \in C^{2}\left(\mathbb{R}^{2}\right)$ are given data, one makes the above observations concerning the coercivity. Likewise to above, coercivity cannot be realized in $C_{\text {per }}^{1}([0,2 \pi])$. The energy space of the bilinear form imposed by the shape Hessian $\nabla^{2} J(\Omega)$ is the Sobolev space $H_{\text {per }}^{1}([0,2 \pi])$, see [12] for the details.

2.3. PDE-Constrained Shape Optimization Problems. We shall consider free elliptic boundary problems as the most illustrational model problem for pdeconstrained shape optimization problems. Let $T \subset \mathbb{R}^{n}$ denote a bounded domain with boundary $\partial T=\Gamma$. Inside the domain $T$ we assume the existence of a simply connected subdomain $S \subset T$ with fixed boundary $\partial S=\Sigma$. We denote the annular domain $T \backslash \bar{S}$ by $\Omega$, see also Figure 2.3 .

We consider the following overdetermined boundary value problem in the annular domain $\Omega$

$$
\begin{aligned}
\Delta u=0 & & \text { in } \Omega, \\
\|\nabla u\|=g & & \text { on } \Gamma, \\
u=0 & & \text { on } \Gamma, \\
u=h & & \text { on } \Sigma,
\end{aligned}
$$

where $g, h>0$ are sufficiently smooth functions such that the shape differentiability of the objective (2.7) is provided up to second order. We like to stress that the positivity of these data implies that $u$ is positive in $\Omega$. Hence, denoting by $\mathbf{n}$ the outer normal at $\Gamma$ with respect to $\Omega$, given by (2.4), there holds the identity

$$
\|\nabla u\| \equiv-\frac{\partial u}{\partial \mathbf{n}} \quad \text { on } \Gamma
$$


since $u$ admits homogeneous Dirichlet data on $\Gamma$.

We arrive at a free boundary problem since the boundary $\Gamma$ is the unknown. In other words, we seek a domain $\Omega$ with fixed boundary $\Sigma$ and unknown boundary $\Gamma$ such that the overdetermined boundary value problem (2.6) is solvable. For the existence of solutions we refer the reader to e.g. [1, 22].

Shape optimization provides an efficient tool to solve such free boundary value problems, cf. [10, 27, 36, 37]. Considering the cost functional

$$
J(\Omega)=\int_{\Omega}\|\nabla u\|^{2}+g^{2} d \mathbf{x}
$$

with underlying state equation

$$
\begin{aligned}
& \Delta u=0 \quad \text { in } \Omega \text {, } \\
& u=0 \quad \text { on } \Gamma \text {, } \\
& u=h \quad \text { on } \Sigma \text {, }
\end{aligned}
$$

the solution of the free boundary problem is equivalent to the shape optimization problem

$$
J(\Omega) \rightarrow \min .
$$

This issues from the necessary condition of a minimizer of the cost functional (2.7), that is,

$$
\nabla J(\Omega)[\mathbf{U}]=\int_{\Gamma}\langle\mathbf{U}, \mathbf{n}\rangle\left\{g^{2}-\left[\frac{\partial u}{\partial \mathbf{n}}\right]^{2}\right\} d \sigma=0
$$

has to be valid for all sufficiently smooth perturbation fields $\mathbf{U}$. Hence, via shape optimization a variational formulation of the condition

$$
\frac{\partial u}{\partial \mathbf{n}}=-g \quad \text { on } \Gamma
$$

is induced. However, a stationary domain $\Omega^{\star}$ of the minimization problem $(2.7),(2.8)$ will be a stable minimum if and only if the shape Hessian is strictly $H^{1 / 2}([0,2 \pi])$ coercive at this domain (see below).

It suffices to consider $S \in C^{0,1}$ but due to a second order boundary perturbation calculus, we have to assume $T \in C^{2, \alpha}$ for some fixed $\alpha \in(0,1)$. We assume likewise to the previous subsection the domain $T$ to be starshaped with respect to $\mathbf{0}$ and apply the same shape calculus. The shape gradient of the cost functional in (2.7) becomes in polar coordinates

$$
\langle\nabla J(\Omega), d r\rangle=\int_{0}^{2 \pi} d r r\left\{g^{2}-\left[\frac{\partial u}{\partial \mathbf{n}}\right]^{2}\right\} d \phi .
$$

The shape Hessian reads in accordance with $[11,12]$ as

$$
\begin{gathered}
\left\langle\nabla^{2} J(\Omega) \cdot d r_{1}, d r_{2}\right\rangle=\int_{0}^{2 \pi} d r_{1} d r_{2}\left\{g^{2}-\left[\frac{\partial u}{\partial \mathbf{n}}\right]^{2}+2 r g\left\langle\nabla g, \mathbf{n}_{0}\right\rangle\right. \\
\left.-\frac{2 r}{\sqrt{r^{2}+r^{\prime 2}}} \frac{\partial u}{\partial \mathbf{n}}\left[r \frac{\partial^{2} u}{\partial \mathbf{n}^{2}}+r^{\prime} \frac{\partial^{2} u}{\partial \mathbf{n} \partial \mathbf{t}}\right]\right\}-2 r d r_{1} \frac{\partial u}{\partial \mathbf{n}} \cdot \frac{\partial d u\left[d r_{2}\right]}{\partial \mathbf{n}} d \phi .
\end{gathered}
$$


Herein, the local shape derivative $d u=d u\left[d r_{2}\right]$ of the state function satisfies

$$
\begin{aligned}
\Delta d u & =0 & & \text { in } \Omega, \\
d u & =0 & & \text { on } \Sigma, \\
d u & =-d r_{2}\left\langle\mathbf{n}_{0}, \mathbf{n}\right\rangle \frac{\partial u}{\partial \mathbf{n}} & & \text { on } \Gamma .
\end{aligned}
$$

Notice that $\partial^{2} u / \partial \mathbf{n}^{2}:=\left\langle\nabla^{2} u \cdot \mathbf{n}, \mathbf{n}\right\rangle$ and $\partial^{2} u /(\partial \mathbf{n} \partial \mathbf{t}):=\left\langle\nabla^{2} u \cdot \mathbf{n}, \mathbf{t}\right\rangle$.

Lemma $2.2([11,21])$. The shape Hessian $\nabla^{2} J(\Omega)$ defines a continuous bilinear form on $H^{1 / 2}([0,2 \pi]) \times H^{1 / 2}([0,2 \pi])$, that is, there exists a constant $c_{S}(\Omega)$ depending only on the actual domain $\Omega$ such that

$$
\left|\nabla^{2} J(\Omega)\left[d r_{1}, d r_{2}\right]\right| \leq c_{S}(\Omega)\left\|d r_{1}\right\|_{H^{1 / 2}([0,2 \pi])}\left\|d r_{2}\right\|_{H^{1 / 2}([0,2 \pi])} .
$$

In accordance with this lemma, we observe that the shape Hessian is a pseudodifferential of order one, i.e. $\nabla^{2} J(\Omega): H^{1 / 2}([0,2 \pi]) \rightarrow H^{-1 / 2}([0,2 \pi])$. In particular, the last term in (2.13) implies that the shape Hessian is a nonlocal operator.

According to [21] the following sufficient criterion concerning the $H^{1 / 2}([0,2 \pi])$ coercivity holds.

Lemma 2.3. The shape Hessian $\nabla^{2} J\left(\Omega^{\star}\right)$ is $H^{1 / 2}([0,2 \pi])$-coercive, that is, there exists a constant $c_{E}>0$ such that

$$
\nabla^{2} J\left(\Omega^{\star}\right)[d r, d r] \geq c_{E}\|d r\|_{H^{1 / 2}([0,2 \pi])}^{2},
$$

if

$$
\kappa+\left[\frac{\partial g}{\partial \mathbf{n}}\right] / g \geq 0 \quad \text { on } \Gamma
$$

In particular, in the case $g \equiv$ const., the shape Hessian is $H^{1 / 2}([0,2 \pi])$-coercive if the boundary $\Gamma^{\star}$ is convex (seen from inside).

The problem under consideration can be viewed as the prototype of a free boundary problem arising in many applications. For example, the growth of anodes in electrochemical processing might be modeled like above with $g, h \equiv 1$.

In the two dimensional exterior magnetic shaping of liquid metals the state equation is an exterior Poisson equation and the uniqueness is ensured by a volume constraint of the domain $\Omega[5,16,31,32]$, cf. the following subsection. However, since the shape functional involves the perimeter, which corresponds to the surface tension of the liquid, the energy space of the Ritz-Galerkin scheme will be $H^{1}([0,2 \pi])$.

The detection of voids or inclusions in $2 \mathrm{~d}$ or $3 \mathrm{~d}$ electrical impedance tomography is slightly different since the roles of $\Sigma$ and $\Gamma$ are interchanged [19, 20, 34]. Particularly, this inverse problem is severely ill-posed, in contrary to the present class of problems. It has been proven in [19] that the shape Hessian is not strictly coercive in any $H^{s}([0,2 \pi])$ for all $s \in \mathbb{R}$.

2.4. Shape Problems with Additional Functional Constraints. We consider the following shape optimization problem

$$
J(\Omega)=\int_{\Omega} j(u, \nabla u, \mathbf{x}) d \mathbf{x} \rightarrow \min ,
$$


subject to $N$ domain respective boundary integral equality constraints

$$
\begin{gathered}
J_{i}(\Omega)=\int_{\Omega} h_{i}(\mathbf{x}) d \mathbf{x}=c_{i}, \quad 1 \leq i \leq M, \\
J_{i}(\Omega)=\int_{\Gamma} g_{i}(\mathbf{x}) d \sigma=c_{i}, \quad M<i \leq N .
\end{gathered}
$$

We suppose that all functionals $J$ and $J_{i}, 1 \leq i \leq N$, are twice Frechét differentiable in a certain Banach space $X$. Moreover, let the Sobolev space $H^{s}$ denote the strongest energy space of the bilinear forms imposed by the shape Hessians of all above shape functionals.

Along the lines of standard optimization theory, one considers the free minimization of the Lagrangian

$$
L\left(\Omega, \lambda_{1}, \ldots, \lambda_{N}\right):=J(\Omega)+\sum_{i=1}^{N} \lambda_{i}\left(J_{i}(\Omega)-c_{i}\right),
$$

if Kuhn-Tucker regularity is provided. Hence, it is well known that the necessary and sufficient optimality condition for a regular local optimal shape $\Omega^{\star}$ reads as

Lemma 2.4. Let $\Omega^{\star} \in X$ satisfy

$$
\nabla L\left(\Omega^{\star}, \lambda_{1}^{\star}, \ldots, \lambda_{N}^{\star}\right)[d r]=0 \quad \text { for all } d r \in X
$$

for certain $\lambda_{i}^{\star} \in \mathbb{R}$. Moreover, define the linearizing cone

$$
Y:=\left\{d r \in X: \nabla J_{i}\left(\Omega^{\star}\right)[d r]=0 \text { for all } 1 \leq i \leq N\right\} \subset X .
$$

Then, $\Omega^{\star}$ is a regular local minimizer of second order if and only if the following coercivity condition is satisfied

$$
\nabla^{2} L\left(\Omega^{\star}, \lambda_{1}^{\star}, \ldots, \lambda_{N}^{\star}\right)[d r, d r] \geq c_{E}\|d r\|_{H^{s}}^{2} \quad \text { for all } d r \in Y .
$$

Consequently, the general concept developed in Section 3 keeps applicable with respect to the Banach space $Y$. We mention that the treatment of inequality constraints is obvious and related modifications are well established in theory.

\section{Approximation Theory in Shape Optimization.}

3.1. Assumptions on the Optimization Problem. Let us first introduce the abstract setting which we will need for our theory. To this end, let $X$ denote a Banach space, where we shall denote the ball $\left\{h \in X:\|r-h\|_{X}<\delta\right\}$ by $B_{\delta}^{X}(r)$.

We consider the following optimization problem in the Banach space $X$

$$
J(r) \rightarrow \min , \quad r \in X .
$$

Herein, $J: X \mapsto \mathbb{R}$ defines a two times continuously differentiable functional, i.e., the gradient $\nabla J(r) \in X^{\star}$ as well as the Hessian $\nabla^{2} J(r) \in L\left(X, X^{\star}\right)$ exist for all $r \in X$, and the mappings $\nabla J(\cdot): X \rightarrow X^{\star}, \nabla J^{2}(\cdot): X \rightarrow L\left(X, X^{\star}\right)$ are continuous.

We assume that in $r^{\star}$ the necessary first order optimality condition holds

$$
\nabla J\left(r^{\star}\right)[d r]=0 \quad \text { for all } d r \in X .
$$


As illustrated in the previous section, we have to take the two norm discrepancy into account, i.e., the coercivity estimate hold only in a weaker Sobolev space $H^{s} \supset X$, $s \geq 0$. Therefore, we shall assume that there is a constant $0<c_{s}$, depending only on the actual variable $r$, such that the continuos bilinear form imposed by the shape Hessian on $X \times X$ extends continuosly to a bilinear form on $H^{s} \times H^{s}$, i.e.,

$$
\left|\nabla^{2} J(r)\left[h_{1}, h_{2}\right]\right| \leq c_{S}(r)\left\|h_{1}\right\|_{H^{s}}\left\|h_{2}\right\|_{H^{s}}, \quad \text { for all } h_{1}, h_{2} \in H^{s},
$$

if $r \in \overline{B_{\delta}^{X}\left(r^{\star}\right)}$. Of course, there exists an absolute constant $C_{S}$, defined by

$$
C_{S}=\max \left\{c_{S}(r): r \in \overline{B_{\delta}^{X}\left(r^{\star}\right)}\right\}
$$

such that $c_{S}(r) \leq C_{S}$ for all $r \in \overline{B_{\delta}^{X}\left(r^{\star}\right)}$. Moreover, we assume that $\nabla^{2} J$ is strongly coercive at $r^{\star}$, that is

$$
\nabla^{2} J\left(r^{\star}\right)[h, h] \geq c_{E}\|h\|_{H^{s}}^{2} \quad \text { for all } h \in H^{s}
$$

for some $c_{E}>0$.

REMARK 3.1. We want to mention in addition that the existence of a continuous extension for the objective $J$ from $X$ to $H^{s}$ is not assumed throughout this paper, since this is not realistic for shape problems in general, see also related remarks in subsection 2.2. That is, J remains only "densely defined" with respect to $H^{s}$, similarly for $\nabla J, \nabla^{2} J$. As it turns out by our investigations, a complete convergence analysis will be possible without assuming such a continuation property.

As a first consequence from our assumptions we conclude the following lemma concerning Lipschitz continuity of the shape gradient with respect to topologies, induced by the coercivity spaces of the shape Hessian.

Lemma 3.2. The gradient is locally Lipschitz as a mapping in the $\left(H^{-s}, H^{s}\right)$ duality $\left(H^{-s}:=\left(H^{s}\right)^{\prime}\right)$, that is

$$
\|\nabla J(r+h)-\nabla J(r)\|_{H^{-s}} \leq C_{S}\|h\|_{H^{s}}
$$

for all $r, r+h \in B_{\delta}^{X}\left(r^{\star}\right)$. Herein, the constant $C_{S}$ is given by (3.1).

Proof. The assertion follows immediately from the following estimate.

$$
|\nabla J(r+h)[d r]-\nabla J(r)[d r]|=\left|\int_{0}^{1}\left\langle\nabla^{2} J(r+t h) \cdot h, d r\right\rangle d t\right| \leq C_{S}\|h\|_{H^{s}}\|d r\|_{H^{s}}
$$

for all $h \in X, d r \in H^{s}$.

Note that the twice differentiability of $J$ provides only the Lipschitz continuity of the shape gradient in the $\left(X^{\star}, X\right)$-duality, i.e.,

$$
\|\nabla J(r+h)-\nabla J(r)\|_{X^{\star}} \leq C_{S}\|h\|_{X} .
$$

for all $r, r+h \in B_{\delta}^{X}\left(r^{\star}\right)$.

3.2. Sufficient Conditions. The above assumptions allow the following statement on the regular local optimality of second order for $r^{\star}$. Whereas this is rather standard from a general optimization point of view, we recall it for convenience.

THEOREM 3.3 (Sufficient second-order optimality condition). Let the necessary condition (A1) hold for a certain $r^{\star} \in X$. Suppose that the bilinear form imposed 
by the shape Hessian satisfies (A2) for all $r \in \overline{B_{\delta}^{X}\left(r^{\star}\right)}$. The domain $r^{\star}$ is a strong regular local optimum of second order

$$
J(r)-J\left(r^{\star}\right) \geq \frac{c_{E}}{4}\left\|r-r^{\star}\right\|_{H^{s}}^{2} \quad \text { for all } r \in B_{\delta}^{X}\left(r^{\star}\right),
$$

if and only if the shape Hessian satisfies the strong coercivity estimate (A3) and the following remainder estimate

$\left|\nabla^{2} J(r)\left[h_{1}, h_{2}\right]-\nabla^{2} J\left(r^{\star}\right)\left[h_{1}, h_{2}\right]\right| \leq \eta\left(\left\|r-r^{\star}\right\|_{X}\right)\left\|h_{1}\right\|_{H^{s}}\left\|h_{2}\right\|_{H^{s}} \quad$ for all $h_{1}, h_{2} \in H^{s}$

holds for all $r \in B_{\delta}^{X}\left(r^{\star}\right)$, where $\eta(t) \rightarrow 0$ if $t \rightarrow 0$.

Proof. For all $r=r^{\star}+h \in B_{\delta}^{X}\left(r^{\star}\right)$ the following Taylor expansion holds

$$
J(r)-J\left(r^{\star}\right)=0+\frac{1}{2} \nabla^{2} J\left(r^{\star}+\xi h\right)[h, h], \quad \xi \in(0,1) .
$$

According to (3.3) and (A4) we infer

$$
\begin{aligned}
J(r)-J\left(r^{\star}\right) & \geq \frac{1}{2} \nabla^{2} J\left(r^{\star}\right)[h, h]-\left|\nabla^{2} J\left(r^{\star}+\xi h\right)[h, h]-\nabla^{2} J\left(r^{\star}\right)\left[h_{1}, h_{2}\right]\right| \\
& \geq \frac{1}{2} \nabla^{2} J\left(r^{\star}\right)[h, h]-\eta\left(\|h\|_{X}\right)\|h\|_{H^{s}}^{2} \\
& \geq \frac{1}{2}\left(c_{E}-\eta\left(\|h\|_{X}\right)\right)\|h\|_{H^{s}}^{2} .
\end{aligned}
$$

Supposing $\delta>0$ to be chosen such that $\eta\left(\|h\|_{X}\right) \leq c_{E} / 2$ for all $h \in B_{\delta}^{X}\left(r^{\star}\right)$, we arrive at

$$
J(r)-J\left(r^{\star}\right) \geq \frac{c_{E}}{4}\left\|r-r^{\star}\right\|_{H^{s}}^{2},
$$

which implies the assertion.

Let us remark that the verification of (A4) turns out to be rather technical in case of pde-constrained shape optimization problems. However, for the presented model problems (A4) has been proven in $[6,7,8]$.

Combining estimate (A4) together with the coercivity (A3) leads to the next lemma.

Corollary 3.4. There exists a $\delta>0$ such that the shape Hessian is strongly coercive in the whole ball $B_{\delta}^{X}\left(r^{\star}\right)$, that is

$$
\nabla^{2} J(r)[h, h] \geq \frac{c_{E}}{2}\|h\|_{H^{s}}^{2} \quad \text { for all } h \in H^{s} .
$$

Proof. Consider $r \in B_{\delta}^{X}\left(r^{\star}\right)$. Then, from (A4) we conclude

$$
\left|\nabla^{2} J(r)[h, h]-\nabla^{2} J\left(r^{\star}\right)[h, h]\right| \leq \eta\left(\left\|r-r^{\star}\right\|_{X}\right)\|h\|_{H^{s}}^{2} \quad \text { for all } h \in H^{s} .
$$

Choosing $\delta>0$ such that $\eta\left(\left\|r-r^{\star}\right\|_{X}\right) \leq c_{E} / 2$ for all $r \in B_{\delta}^{X}(0)$ the assertion follows immediately from (3.3) and

$$
\nabla^{2} J(r)[h, h] \geq \nabla^{2} J\left(r^{\star}\right)[h, h]-\eta\left(\left\|r-r^{\star}\right\|_{X}\right)\|h\|_{H^{s}}^{2} \geq \frac{c_{E}}{2}\|h\|_{H^{s}}^{2} .
$$

Remark 3.5. Combining the continuity of the shape Hessian (A2) with (3.1) we conclude in addition the estimate

$$
\frac{c_{E}}{4}\left\|r-r^{\star}\right\|_{H^{s}}^{2} \leq J(r)-J\left(r^{\star}\right) \leq \frac{C_{S}}{2}\left\|r-r^{\star}\right\|_{H^{s}}^{2}
$$

for all $r \in B_{\delta}^{X}\left(r^{\star}\right)$. 
3.3. Ritz-Galerkin Discretization. We shall consider a Ritz-Galerkin scheme to solve the necessary condition (A1), i.e., we replace the given infinite dimensional optimization problem by a finite dimensional problem. The trial space should provide some additional regularity in order to approximate functions in $X$. To that end, we introduce an appropriate Hilbert space $H^{k} \subset X$, continuously embedded in $X$

$$
\|r\|_{X} \leq c_{H^{k} \rightarrow X}\|r\|_{H^{k}} \quad \text { for all } r \in H^{k} .
$$

Then, we shall consider a sequence of nested finite dimensional trial spaces

$$
V_{0} \subset V_{1} \subset \ldots \subset V_{N} \subset \ldots \subset H^{k} \subset X, \bigcap_{N \geq 0} V_{N}=V_{0}, \quad \varlimsup_{N \geq 0} V_{N} H^{k}=H^{k},
$$

providing the following inverse estimate

$$
\left\|r_{N}\right\|_{H^{k}} \leq E(N)\left\|r_{N}\right\|_{H^{s}} \text { for all } r_{N} \in V_{N} .
$$

Moreover, we assume that there exists an $L>k$ such that the following approximation property holds

$$
\inf _{r_{N} \in V_{N}}\left\|r-r_{N}\right\|_{H^{s}}=o\left(\frac{1}{E(N)}\right)\|r\|_{H^{\ell}} \quad \text { if } r \in H^{\ell}(k<\ell \leq L) .
$$

Herein, the Landau symbol $g(x)=o(f(x))$ means that $\lim _{x \rightarrow \infty} g(x) / f(x)=0$.

Remark 3.6. Suppose $X=C^{2, \alpha}([0,1])$ for some $\alpha \in(0,1)$, then the Sobolev space $H^{k}([0,1])$ with $3 \geq k>2+\alpha$ provides a continuous embedding in accordance with (V1). Choosing $V_{N} \subset C^{2,1}([0,1])$ as the space of smoothest cubic splines on the uniform subdivision with step width $h_{N}:=2^{-N} / 4$, we have the approximation property

$$
\inf _{r_{N} \in V_{N}}\left\|r-r_{N}\right\|_{H^{s}} \lesssim h_{N}^{\ell-s}\|r\|_{H^{\ell}} \quad \text { if } r \in H^{\ell}(k<\ell \leq 4)
$$

uniformly in $N$ provided that $s<k$. The inverse estimate reads as

$$
\left\|r_{N}\right\|_{H^{k}} \lesssim h_{N}^{k-s}\left\|r_{N}\right\|_{H^{s}} \quad \text { for all } r_{N} \in V_{N}
$$

uniformly in $N$ provided that $k \leq s$. Hence, we conclude that the trial spaces $\left(V_{N}\right)_{N \geq 0}$ satisfy (V2)-(V4).

The Ritz-Galerkin scheme reads as follows. In order to solve

$$
J\left(r_{N}\right) \rightarrow \min , \quad r_{N} \in V_{N},
$$

one seeks an approximate solution $r_{N} \in V_{N}$ such that the discretized necessary condition

$$
\nabla J\left(r_{N}\right)\left[q_{N}\right]=0
$$

holds for all $q_{N} \in V_{N}$.

There are different strategies to find $r_{N} \in V_{N}$ such that (3.5) holds. In general, supposed that $r_{N}$ has $N$ degrees of freedom, i.e., there exist $\varphi_{1}, \ldots, \varphi_{N}$ such that

$$
V_{N}=\operatorname{span}\left\{\varphi_{i}: i=1, \ldots, \phi_{N}\right\},
$$


one makes the ansatz $r_{N}=\sum_{i=1}^{N} r_{i} \phi_{i}$ and considers an iterative scheme

$$
\mathbf{r}^{(n+1)}=\mathbf{r}^{(n)}-h^{(n)} \mathbf{M}^{(n)} \mathbf{G}^{(n)},
$$

where $h^{(n)}$ is a suitable step width and

$$
\mathbf{r}^{(n)}=\left(r_{i}^{(n)}\right)_{i=1, \ldots, N}, \quad \mathbf{G}:=\left(\nabla J\left(r_{N}^{(n)}\right)\left[\phi_{i}\right]\right)_{i=1, \ldots, N} .
$$

First order methods are the gradient method $\left(\mathbf{M}^{(n)}:=\mathbf{I}\right)$ or the quasi Newton method where $\mathbf{M}^{(n)}$ denotes a suitable approximation to the inverse shape Hessian. Choosing

$$
\mathbf{M}^{(n)}:=\left(\nabla^{2} J\left(r_{N}^{(n)}\right)\left[\phi_{i}, \phi_{j}\right]\right)_{i, j=1, \ldots, N}^{-1}
$$

we arrive at the Newton method, which converges much faster compared to the first order methods, see [15] for example.

3.4. Existence of Approximate Solutions. We will consider the existence of solutions of (3.5) and the question of the accuracy of approximate solutions $r_{N}^{\star}$. Since the solutions of (3.5) are only stationary points, it is reasonable to consider only local optimization problems. Therefore, we replace the global problems $(P)$ and $\left(P_{N}\right)$ by the local optimization problem

$$
J(r) \rightarrow \min , \quad r \in \overline{B_{\delta}^{X}\left(r^{\star}\right)}
$$

and its discrete variant

$$
J\left(r_{N}\right) \rightarrow \min , \quad r_{N} \in V_{N} \cap \overline{B_{\delta}^{X}\left(r^{\star}\right)} . \quad\left(P_{N}^{\delta}\right)
$$

The solution of $\left(P^{\delta}\right)$ is $r^{\star}$ since $J$ is strictly coercive on the convex set $\overline{B_{\delta}^{X}\left(r^{\star}\right)}$ Likewise, $\left(P_{N}^{\delta}\right)$ admits always a solution $r_{N}^{\star} \in V_{N} \cap \overline{B_{\delta}^{X}\left(r^{\star}\right)}$. Any point $r_{N}^{\star} \in V_{N} \cap$ $\overline{B_{\delta}^{X}\left(r^{\star}\right)}$ satisfying (3.5) is a local regular optimizer of second order. Moreover, the coercivity implies the uniqueness of $r_{N}^{\star}$. Nevertheless, for a complete convergence analysis, we have to ensure that $r_{N}^{\star}$ is an interior point of the convex set $V_{N} \cap \overline{B_{\delta}^{X}\left(r^{\star}\right)}$, i.e.,

$$
\left\|r_{N}^{\star}-r^{\star}\right\|_{X}<\delta
$$

Theorem 3.7. Let (A1)-(A4) and (V1)-(V4) hold. Then, if $r^{\star} \in H^{\ell}$ for some $\ell>k$, there exists an $N_{0}$ such that

$$
r_{N}^{\star} \in V_{N} \cap B_{\delta}^{X}\left(r^{\star}\right) \quad \text { for all } N \geq N_{0} .
$$

Proof. We split the proof into four parts.

(i). We define $P_{N}: L^{2} \rightarrow V_{N}$ as the $L^{2}$-orthogonal projection onto $V_{N}$. Then, by our assumptions (V1),(V2) we have

$$
\left\|P_{N}\left(r^{\star}\right)-r^{\star}\right\|_{X} \leq c_{H^{k} \rightarrow X}\left\|P_{N}\left(r^{\star}\right)-r^{\star}\right\|_{H^{k}} \lesssim \inf _{r_{N} \in V_{N}}\left\|r_{N}-r^{\star}\right\|_{H^{k}} \stackrel{N \rightarrow \infty}{\longrightarrow} 0
$$

and likewise by (V4)

$$
\left\|P_{N}\left(r^{\star}\right)-r^{\star}\right\|_{H^{s}} \lesssim \inf _{r_{N} \in V_{N}}\left\|r_{N}-r^{\star}\right\|_{H^{s}} \stackrel{N \rightarrow \infty}{\longrightarrow} 0 .
$$


Hence, we deduce that there exists an $N_{0}$ such that $V_{N} \cap \overline{B_{\delta}^{X}}\left(r^{\star}\right) \neq \emptyset$ for all $N \geq N_{0}$. Without loss of generality we assume that $N_{0}=0$.

(ii). Recall that

$$
\begin{gathered}
J\left(r^{\star}\right)=\inf \left\{J(r): r \in \overline{B_{\delta}^{X}\left(r^{\star}\right)}\right\}, \\
J\left(r_{N}^{\star}\right)=\inf \left\{J\left(r_{N}\right): r_{N} \in V_{N} \cap \overline{B_{\delta}^{X}\left(r^{\star}\right)}\right\},
\end{gathered}
$$

and define $J_{\delta}(N) \geq J\left(r_{N}^{\star}\right) \geq J\left(r^{\star}\right)$ via

$$
J_{\delta}(N):=\inf \left\{J\left(r_{N}\right): r_{N} \in V_{N} \cap \partial B_{\delta}^{X}\left(r^{\star}\right)\right\} .
$$

Since $J\left(P_{N}\left(r^{\star}\right)\right) \geq J\left(r_{N}^{\star}\right)$, we conclude the assertion $\left\|r_{N}^{\star}-r^{\star}\right\|_{X}<\delta$ if we can proof

$$
J_{\delta}(N)>J\left(P_{N}\left(r^{\star}\right)\right) \text { for all } N \geq N_{0} .
$$

On the one hand, (3.4) implies

$$
J\left(P_{N}\left(r^{\star}\right)\right)-J\left(r^{\star}\right) \leq \frac{C_{S}}{2}\left\|r^{\star}-P_{N}\left(r^{\star}\right)\right\|_{H^{s}}^{2} .
$$

On the other hand, introducing the quantity

$$
\begin{aligned}
F_{\delta}^{X}(N) & :=\inf \left\{\left\|r_{N}-r^{\star}\right\|_{H^{s}}: r_{N} \in V_{N} \cap \partial B_{\delta}^{X}\left(r^{\star}\right)\right\} \\
& =\inf \left\{\left\|r_{N}-r^{\star}\right\|_{H^{s}}: r_{N} \in V_{N} \backslash B_{\delta}^{X}\left(r^{\star}\right)\right\}
\end{aligned}
$$

we derive from (3.4)

$$
J_{\delta}(N)-J\left(r^{\star}\right) \geq \frac{c_{E}}{4} F_{\delta}^{X}(N)^{2} .
$$

Combining (3.8) and (3.9), the inequality

$$
\left\|r^{\star}-P_{N}\left(r^{\star}\right)\right\|_{H^{s}}<C^{\star} \cdot F_{\delta}^{X}(N), \quad C^{\star}:=\sqrt{\frac{c_{E}}{2 C_{S}}},
$$

implies (3.7) and, thus, $\left\|r_{N}^{\star}-r^{\star}\right\|_{X}<\delta$.

(iii). We shall establish a relation between $F_{\delta}^{X}(N),\left\|r^{\star}-P_{N}\left(r^{\star}\right)\right\|_{H^{s}}$, and $E(N)$ from the inverse estimate (V3). For sake of simplicity, we assume without loss of generality the constant $c_{H^{k} \rightarrow X}$ from (V1) to be less than one such that

$$
B_{\delta}^{H^{k}}\left(r^{\star}\right) \subset B_{\delta}^{X}\left(r^{\star}\right) .
$$

Introducing

$$
\begin{aligned}
F_{\delta}^{H^{k}}(N) & :=\inf \left\{\left\|r_{N}-r^{\star}\right\|_{H^{s}}: r_{N} \in V_{N} \cap \partial B_{\delta}^{H^{k}}\left(r^{\star}\right)\right\} \\
& =\inf \left\{\left\|r_{N}-r^{\star}\right\|_{H^{s}}: r_{N} \in V_{N} \backslash B_{\delta}^{H^{k}}\left(r^{\star}\right)\right\},
\end{aligned}
$$

there follows from (3.11) the relation

$$
F_{\delta}^{H^{k}}(N) \leq F_{\delta}^{X}(N) .
$$

We shall now compute a lower bound for $F_{\delta}^{H^{k}}(N)$. From

$$
\left\|r_{N}-P_{N}\left(r^{\star}\right)\right\|_{H^{s}}-\left\|P_{N}\left(r^{\star}\right)-r^{\star}\right\|_{H^{s}} \leq\left\|r_{N}-r^{\star}\right\|_{H^{s}}
$$


one infers the inequality

$$
F_{\delta}^{H^{k}}(N) \geq \inf \left\{\left\|r_{N}-P_{N}\left(r^{\star}\right)\right\|_{H^{s}}: r_{N} \in V_{N} \backslash B_{\delta}^{H^{k}}\left(r^{\star}\right)\right\}-\left\|P_{N}\left(r^{\star}\right)-r^{\star}\right\|_{H^{s}} .
$$

We choose $N_{0}$ sufficiently large such that

$$
\left\|P_{N}\left(r^{\star}\right)-r^{\star}\right\|_{H^{s}} \leq \delta / 2 \text { for all } N \geq N_{0} .
$$

for all $N \geq N_{0}$. Then, it holds $B_{\delta / 2}^{H^{k}}\left(P_{N}\left(r^{\star}\right)\right) \subset B_{\delta}^{H^{k}}\left(r^{\star}\right)$ and we arrive at

$$
\begin{aligned}
\inf \{\| & \left.r_{N}-P_{N}\left(r^{\star}\right) \|_{H^{s}}: r_{N} \in V_{N} \backslash B_{\delta}^{H^{k}}\left(r^{\star}\right)\right\} \\
& \geq \inf \left\{\left\|r_{N}-P_{N}\left(r^{\star}\right)\right\|_{H^{s}}: r_{N} \in V_{N} \backslash B_{\delta / 2}^{H^{k}}\left(P_{N}\left(r^{\star}\right)\right)\right\} \\
& \geq \inf _{r_{N} \in V_{N}}\left\{\left\|r_{N}\right\|_{H^{s}}:\left\|r_{N}\right\|_{H_{k}}=\delta / 2\right\} \\
& \geq \frac{\delta}{2 E(N)} .
\end{aligned}
$$

Inserting this estimate into (3.12), we deduce

$$
F_{\delta}^{X}(N) \geq F_{\delta}^{H^{k}}(N) \geq \frac{\delta}{2 E(N)}-\left\|P_{N}\left(r^{\star}\right)-r^{\star}\right\|_{H^{s}} \quad \text { for all } N \geq N_{0} .
$$

(iv). Observing

$$
\left\|P_{N}\left(r^{\star}\right)-r^{\star}\right\|_{H^{s}} \lesssim \inf _{r_{N} \in V_{N}}\left\|r_{N}-r^{\star}\right\|_{H^{s}}
$$

we infer from (V4) that we can increase $N_{0}$ such that

$$
\left\|P_{N}\left(r^{\star}\right)-r^{\star}\right\|_{H^{s}}<\frac{\delta}{2 E(N)} \cdot \frac{C^{\star}}{C^{\star}+1} \quad \text { for all } N \geq N_{0} .
$$

Thus, we arrive at

$$
\left\|P_{N}\left(r^{\star}\right)-r^{\star}\right\|_{H^{s}}<C^{\star}\left(\frac{\delta}{2 E(N)}-\left\|P_{N}\left(r^{\star}\right)-r^{\star}\right\|_{H^{s}}\right)<C^{\star} F_{\delta}^{X}(N),
$$

that is (3.10), for all $N \geq N_{0}$, which finishes the proof according to part (ii). $\square$

REMARK 3.8. Obviously, by means of standard optimization theory, (3.3) together with (3.5), imply well-posedness of the finite dimensional optimization problems, that is, the convergence

$$
r_{N}^{(n)} \rightarrow r_{N}^{\star}, \quad \text { if } n \rightarrow \infty
$$

of the iterative scheme (3.6) is provided.

3.5. Convergence. The above theorem ensures the existence of the approximate solution $r_{N}^{\star}$ of the finite dimensional problems $\left(P_{N}^{\delta}\right)$ if $N$ is sufficiently large. The next theorem estimates the distance $\left\|r_{N}^{\star}-r^{\star}\right\|_{H^{s}}$.

THEOREM 3.9. The approximate solution $r_{N}^{\star}$ of the finite dimensional problem $\left(P_{N}^{\delta}\right)$ satisfies the error estimate

$$
\left\|r_{N}^{\star}-r^{\star}\right\|_{H^{s}} \leq \frac{C_{S}}{c_{E}} \inf _{r_{N} \in V_{N}}\left\|r_{N}-r^{\star}\right\|_{H^{s}}
$$


uniformly with the number of unknowns $N$.

Proof. For sake of clearness in representation let $\langle\cdot, \cdot\rangle$ denote the continuous extension of the $L^{2}$-inner product onto $H^{-s} \times H^{s}$.

On the one hand, observing (3.2), Galerkin orthogonality implies

$$
\begin{aligned}
\left\langle\nabla J\left(r_{N}^{\star}\right)-\nabla J\left(r^{\star}\right), r_{N}^{\star}-r^{\star}\right\rangle & =\left\langle\nabla J\left(r_{N}^{\star}\right)-\nabla J\left(r^{\star}\right), r_{N}-r^{\star}\right\rangle \\
& \leq C_{S}\left\|r_{N}^{\star}-r^{\star}\right\|_{H^{s}}\left\|r_{N}-r^{\star}\right\|_{H^{s}}
\end{aligned}
$$

for all $r_{N} \in V_{N}$. On the other hand, introducing

$$
j(t):=\left\langle\nabla J\left(t r_{N}^{\star}-(1-t) r^{\star}\right), r_{N}^{\star}-r^{\star}\right\rangle,
$$

we derive the estimate

$$
\begin{aligned}
&\left\langle\nabla J\left(r_{N}^{\star}\right)-\nabla\right.\left.J\left(r^{\star}\right), r_{N}^{\star}-r^{\star}\right\rangle=j(1)-j(0)=\int_{0}^{1} j^{\prime}(t) d t= \\
& \quad=\int_{0}^{1}\left\langle\nabla^{2} J\left(r_{t}\right) \cdot\left(r_{N}^{\star}-r^{\star}\right), r_{N}^{\star}-r^{\star}\right\rangle d t \geq c_{E}\left\|r_{N}^{\star}-r^{\star}\right\|_{H^{s}}^{2} .
\end{aligned}
$$

Combining both estimates yields

$$
\left\|r_{N}^{\star}-r^{\star}\right\|_{H^{s}}^{2} \leq \frac{C_{S}}{c_{E}}\left\|r_{N}^{\star}-r^{\star}\right\|_{H^{s}}\left\|r_{N}-r^{\star}\right\|_{H^{s}}
$$

for all $r_{N} \in V_{N}$, which is equivalent to the assertion.

Of course, from this theorem one can determine the rate of convergence, if one estimates $\inf _{r_{N} \in V_{N}}\left\|r_{N}-r^{\star}\right\|_{H^{s}}$.

\section{Numerical results.}

4.1. An Unconstrained Shape Optimization Problem. For comparison reasons we shall employ model problems where the solution is known analytically. To this end, we choose the shape optimization problem (2.3) based on the domain integral

$$
J(\Omega)=\int_{\Omega}\left(\frac{x^{2}}{8}+\frac{y^{2}}{4}-2\right) d \mathbf{x}
$$

as our first numerical example. In accordance to Subsection 2.2, the solution is the ellipse centered in $\mathbf{0}$ with semi-axes $2 \sqrt{2}$ and 2 .

The numerical setting is as follows. We subdivide the parameter interval $[0,2 \pi]$ equidistantly into $N$ intervals. With respect to this subdivision, the radial function $r \in C_{\text {per }}^{1}([0,2 \pi])$ is then approximated periodically by $N$ cubic B-splines $B_{i}^{3}, i=$ $1, \ldots, N$, that is

$$
r_{N}=\sum_{i=1}^{N} a_{i} B_{i}^{3} \in C_{\text {per }}^{2,1}([0,2 \pi]) .
$$

We employ a Newton method to solve the necessary condition $\nabla J(\Omega) \equiv 0$ iteratively, using the circle with radius 2 as initial guess.

Since the energy space for the shape Hessian is $L^{2}([0,2 \pi])$, we measure the $L^{2}$ norm of the approximation error

$$
\left\|r-r_{N}\right\|_{L^{2}([0,2 \pi])}=\sqrt{\int_{0}^{2 \pi}\left|r-r_{N}\right|^{2} d \phi} .
$$

The measurements are visualized in Figure 4.1. We observe the as predicted the rate of convergence $N^{-4}$, indicated by the dashed line. 


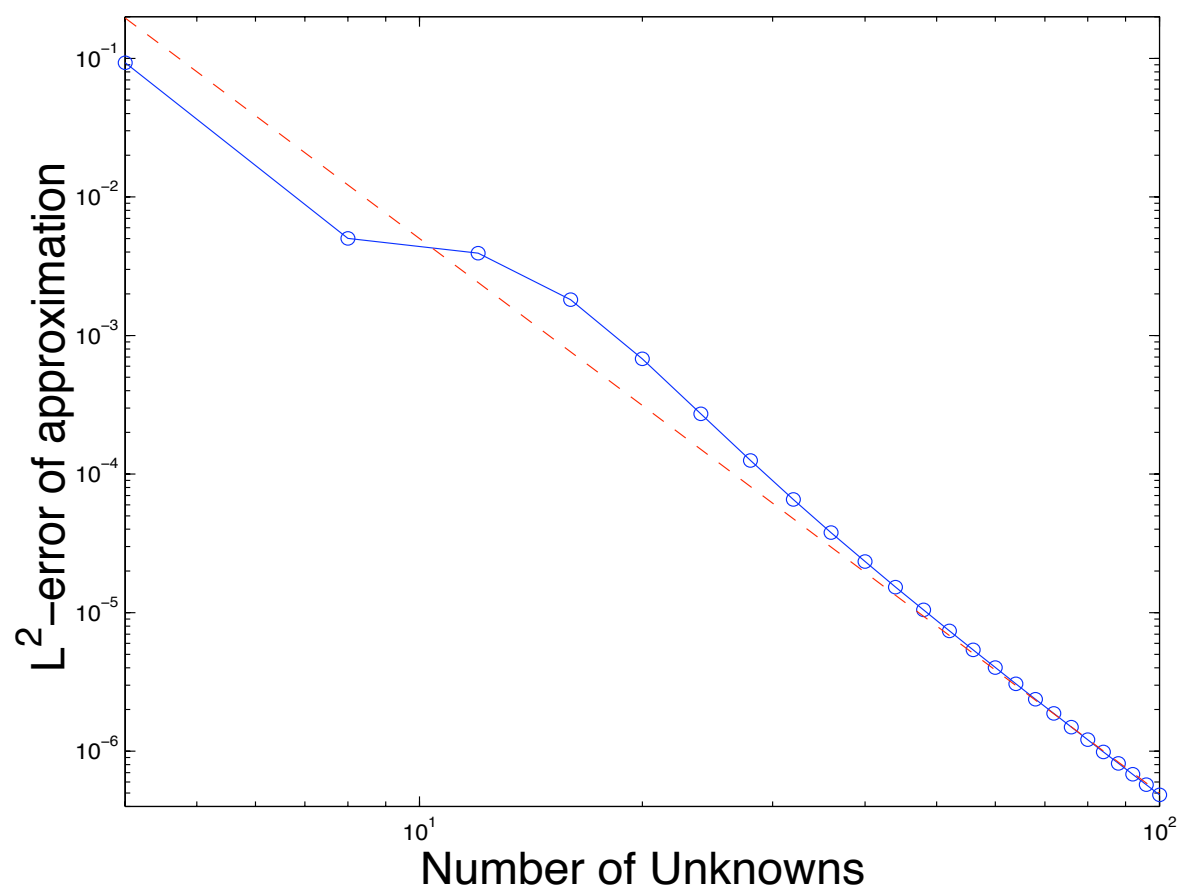

FIG. 4.1. $L^{2}$-error of the approximate solution.

4.2. A Constrained Shape Optimization Problem. We consider next a cylindric circular bar which is homogeneous and isotropic with a planar, simply connected cross section $\Omega \in \mathbb{R}^{2}$. We follow Banichuk and Karihaloo [2] but normalize the shear modulus $G=1$ and the elastic modulus $E=1$. We want to solve the problem of maximizing the torsional rigidity of the bar subject to given equality constraints on the stiffness rigidity and the volume.

First, we briefly recall the mathematical formulation of the quantities. The torsional rigidity is calculated by

$$
T(\Omega)=2 \int_{\Omega} u(\mathbf{x}) d \mathbf{x}
$$

where the stress function $u=u(\Omega)$ satisfies

$$
\begin{aligned}
-\Delta u=2 & \text { in } \Omega, \\
u=0 & \text { on } \Gamma .
\end{aligned}
$$

The bending rigidity with respect to a fixed barycentre in the origin is given by

$$
B(\Omega)=\int_{\Omega} y^{2} d \mathbf{x}
$$

The volume of the domain and its (simplified) barycentre coordinates read as

$$
V(\Omega)=\int_{\Omega} d \mathbf{x}, \quad S_{x}(\Omega)=\int_{\Omega} x d \mathbf{x}, \quad S_{y}(\Omega)=\int_{\Omega} y d \mathbf{x} .
$$


Choosing $B_{0}=\sqrt{2} \pi / 4, V_{0}=\pi$, then the necessary condition is fulfilled by the ellipse with semiaxes $h_{x}=2^{-1 / 4}$ and $h_{y}=2^{1 / 4}$. The associated Lagrange multipliers are $\lambda_{B}=-4 / 9, \lambda_{V}=8 \sqrt{2} / 9$, and $\lambda_{S_{x}}=\lambda_{S_{y}}=0$, cf. [2]. Despite the fact the sufficient optimality conditions have not been proved yet, our experiences indicated coercivity with the energy space $H^{1 / 2}$, cf. [14, 15, 17].

¿From the identity

$$
T(\Omega)=\int_{\Omega}\|\nabla u(\mathbf{x})\|^{2} d \mathbf{x}
$$

we deduce that $\nabla T(\Omega)[d r]$ and $\nabla^{2} T(\Omega)\left[d r_{1}, d r_{2}\right]$ are given as in (2.12) and (2.13) with $g \equiv 0$ and

$$
\begin{aligned}
\Delta d u & =0 & & \text { in } \Omega, \\
d u & =-d r_{2}\left\langle\mathbf{n}_{0}, \mathbf{n}\right\rangle \frac{\partial u}{\partial \mathbf{n}} & & \text { on } \Gamma .
\end{aligned}
$$

The computation of the other gradients and Hessians is straightforward, cf. $[14,15]$ for the details. The energy space of the coercivity estimate is obviously $H^{1 / 2}([0,2 \pi])$.

The radial function $r$ is approximated likewise to our first example by periodic cubic Splines on the interval $[0,2 \pi]$. In order to solve the discretized constrained shape optimization problem, we shall find the stationary points of the following augmented Lagrange functional

$$
L_{\alpha}(\Omega, \lambda):=-T(\Omega)+\boldsymbol{\lambda}^{T}\left[\begin{array}{c}
B(\Omega)-B_{0} \\
V(\Omega)-V_{0} \\
S_{x}(\Omega) \\
S_{y}(\Omega)
\end{array}\right]+\frac{\alpha}{2}\left\|\left[\begin{array}{c}
B(\Omega)-B_{0} \\
V(\Omega)-V_{0} \\
S_{x}(\Omega) \\
S_{y}(\Omega)
\end{array}\right]\right\|^{2}
$$

where $\boldsymbol{\lambda}:=\left(\lambda_{B}, \lambda_{V}, \lambda_{S_{x}}, \lambda_{S_{y}}\right)$ and $\alpha>0$ is an appropriate chosen penalty parameter. The optimization algorithm for the Augmented Lagrangian method reads as follows:

- initialization: choose initial guesses $\boldsymbol{\lambda}^{(0)}$ and $\Omega^{(0)}$ for $\boldsymbol{\lambda}^{\star}$ and $\Omega^{\star}$, respectively,

- inner iteration: solve $\Omega^{(n+1)}:=\operatorname{argmin} L_{\alpha}\left(\Omega, \lambda^{(n)}\right)$ with initial guess $\Omega^{(n)}$,

- outer iteration: update

$$
\lambda^{(n+1)}:=\lambda^{(n)}-\alpha\left[\begin{array}{c}
B\left(\Omega^{(n+1)}\right)-B_{0} \\
V\left(\Omega^{(n+1)}\right)-V_{0} \\
S_{x}\left(\Omega^{(n+1)}\right) \\
S_{y}\left(\Omega^{(n+1)}\right)
\end{array}\right] .
$$

In the inner iteration, we employ a Newton scheme combined with a quadratic linesearch. Instead of the first order update rule described above, we use a second order Lagrange multiplier update introduced in [29], see also [17]. The state equation is solved by using a boundary element method, cf. $[14,15]$ for the details. Notice that about 2000 boundary elements are required to solve the state equation sufficiently accurate if we discretize the free boundary by $N=100 \mathrm{~B}$-splines.

According to our convergence result we shall observe the rate of convergence

$$
\left\|r-r_{N}\right\|_{H^{1 / 2}([0,2 \pi])} \lesssim N^{-3.5}\|r\|_{H^{4}([0,2 \pi])} .
$$

We measure this norm via the approximation

$$
\left\|r-r_{N}\right\|_{H^{1 / 2}([0,2 \pi])} \sim \sqrt{\int_{0}^{2 \pi}\left|r-r_{N}\right|\left|r^{\prime}-r_{N}^{\prime}\right| d \phi} .
$$




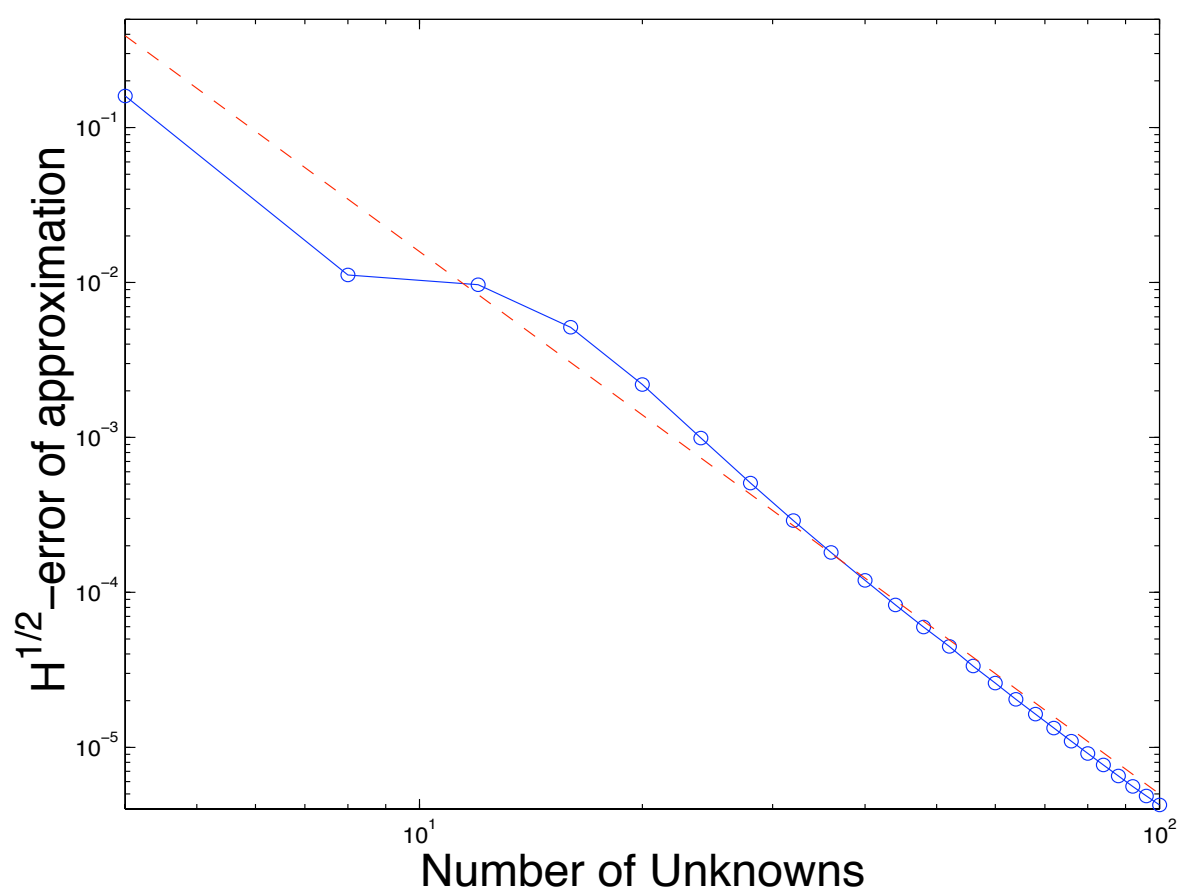

FIG. 4.2. $H^{1 / 2}$-error of the approximate solution.

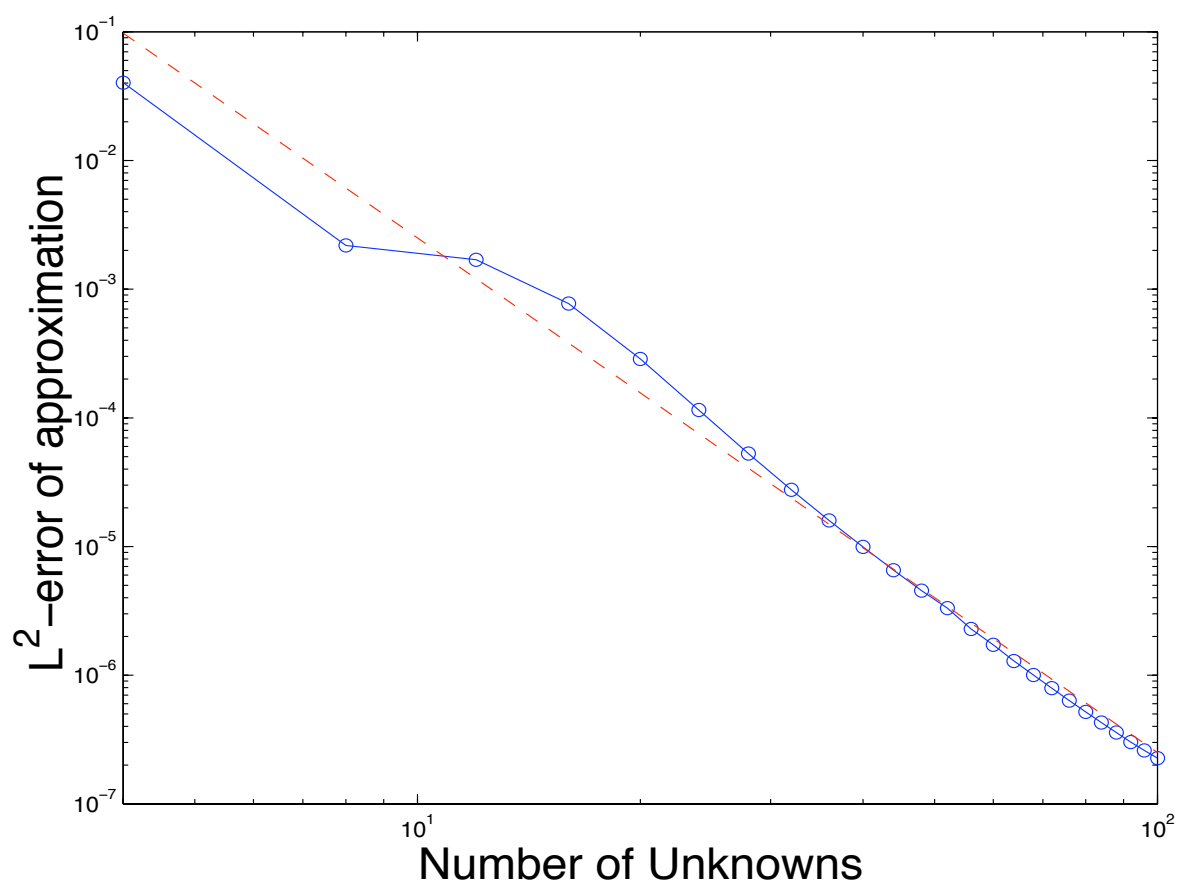

FIG. 4.3. $L^{2}$-error of the approximate solution. 
The results are presented in Figure 4.2. As predicted, the error decreases like $N^{-3.5}$, which is indicated by the dashed line.

In addition we measured also the $L^{2}$-norm of the approximation error, visualized in Figure 4.2. In fact, even though we have not proven the Aubin-Nitsche trick, we observe the higher rate of convergence $N^{-4}$, indicated by the dashed line.

4.3. Concluding remarks. In the present paper we established a complete convergence analysis for approximate solutions of shape optimization problems. In particular, we incorporated the two norm discrepancy. We presented numerical results which verify our analysis quite well. We like to point out that our analysis applies also to $p$-discretizations of the parametrization, for example finite dimensional Fourier sequences for the discretization of the radial function. For several applications we refer to $[5,14,15,16]$, see also $[18,20]$ for related problems in $3 \mathrm{D}$. The treatment of the fully discretized optimization problem is straighforward by incorporating the error estimates concerned with the numerical solution of the state equation, see e.g. $[14,15,18]$.

\section{REFERENCES}

[1] H.W. Alt and L.A. Caffarelli. Existence and regularity for a minimum problem with free boundary. J. reine angew. Math., 325:105-144, 1981.

[2] N.V. Banichuk and B.L. Karihaloo. Minimum-weight design of multi-purpose cylindrical bars. International Journal of solids and Structures, 12:267-273, 1976.

[3] E. Casas, F. Tröltzsch and A. Unger. Second order sufficient optimality conditions for a nonlinear elliptic boundary control problem. Z. Anal. Anwend., 15:687-707, 1996.

[4] E. Casas, F. Tröltzsch and A. Unger. Second order sufficient optimality conditions for some state-constrained control problems of semilinear elliptic equations. SIAM J. Control Optimization 38:1369-1391, 2000.

[5] O. Colaud and A. Henrot. Numerical approximation of a free boundary problem arising in electromagnetic shaping. SIAM J. Numer. Anal.. 31:1109-1127, 1994.

[6] M. Dambrine and M. Pierre. About stability of equilibrium shapes. M2AN, Math. Model. Numer. Anal. 34:811-834, 2000.

[7] M. Dambrine. Hessiennes de forme et stabilité des formes critiques. thesis (in french), ENS Cachan Bretagne, Rennes, 2000.

[8] M. Dambrine. On variations of the shape Hessian and sufficient conditions for the stability of critical shapes. RACSAM, Rev. R. Acad. Cien. Serie A. Mat. 96:95-121, 2002.

[9] M. Delfour and J.-P. Zolesio. Velocity method and Lagrangian formulation for the computation of the shape Hessian. SIAM J. Control Optimization, 29:1414-1442, 1991.

[10] M. Delfour and J.-P. Zolesio. Shapes and Geometries. SIAM, Philadelphia, 2001.

[11] K. Eppler. Boundary integral representations of second derivatives in shape optimization. Discussiones Mathematicae (Differential Inclusion Control and Optimization), 20:63-78, 2000.

[12] K. Eppler. Optimal shape design for elliptic equations via BIE-methods. J. of Applied Mathematics and Computer Science, 10:487-516, 2000.

[13] K. Eppler. Second derivatives and sufficient optimality conditions for shape functionals. Control and Cybernetics. 29:485-512, 2000.

[14] K. Eppler and H. Harbrecht. Numerical solution of elliptic shape optimization problems using wavelet-based BEM. Optim. Methods Softw., 18:105-123, 2003.

[15] K. Eppler and H. Harbrecht. 2nd Order Shape Optimization using Wavelet BEM. Preprint 06-2003, TU Berlin, 2003. to appear in Optim. Methods Softw.

[16] K. Eppler and H. Harbrecht. Exterior Electromagnetic Shaping using Wavelet BEM. Technical Report 13-2003, Math. Meth. Appl. Sci. 28:387-405 (2005).

[17] K. Eppler and H. Harbrecht. Second order Lagrange multiplier approximation for constrained shape optimization problems. in Shape Optimization and Optimal Design, (Lecture Notes in Pure and Applied Mathematics), edited by J. Cagnol et. al., Marcel Dekker, 2005.

[18] K. Eppler and H. Harbrecht. Fast wavelet BEM for 3d electromagnetic shaping. Bericht 039, Berichtsreihe des Mathematischen Seminars der Christian-Albrechts-Universit zu Kiel, 2003. to appear in Appl. Numer. Math. 
[19] K. Eppler and H. Harbrecht. A regularized Newton method in electrical impedance tomography using shape Hessian information. WIAS-Preprint No. 943, WIAS Berlin, 2004. to appear in Control \& Cybernetics.

[20] K. Eppler and H. Harbrecht. Shape optimization in 3D electrical impedance tomography. WIAS-Preprint 963, WIAS Berlin, 2004. submitted to Proceeding of IFIP TC 7.2 conference.

[21] K. Eppler and H. Harbrecht. Efficient treatment of stationary free boundary problems. WIASPreprint 965, WIAS Berlin, 2004. submitted to Appl. Numer. Math.

[22] M. Flucher and M. Rumpf. Bernoulli's free-boundary problem, qualitative theory and numerical approximation. J. reine angew. Math., 486:165-204, 1997.

[23] H. Goldberg and F. Tröltzsch. Second order optimality conditions for a class of control problems governed by nonlinear integral equations with application to parabolic boundary control. Optimization 20: 687-698, 1989.

[24] H. Goldberg and F. Tröltzsch. Second-order optimality conditions for a class of nonlinear parabolic boundary control problems. SIAM J. Control Optimization 31: 1007-1025, 1993.

[25] J. Hadamard. Lessons on Calculus of Variations. Gauthier-Villiars, Paris, 1910 (in French).

[26] J. Haslinger and P. Neitaanmäki. Finite element approximation for optimal shape, material and topology design, 2nd edition. Wiley, Chichester, 1996.

[27] J. Haslinger, T. Kozubek, K. Kunisch and G. Peichl. Shape optimization and fictitious domain approach for solving free boundary value problems of Bernoulli type. Computational Optimization and Applications, 26:231-251, 2003.

[28] A.M. Khludnev and J. Sokolowski. Modelling and control in solid mechanics. Birkhäuser, Basel, 1997.

[29] K. Mårtensson. A new approach to constrained function optimization. J. of Optimization Theory and Application. 12: 531-554, 1973.

[30] F. Murat and J. Simon. Étude de problémes d'optimal design. in Optimization Techniques, Modeling and Optimization in the Service of Man, edited by J. Céa, Lect. Notes Comput. Sci. 41, Springer-Verlag, Berlin, 54-62 (1976).

[31] A. Novruzi and J.R. Roche. Second derivatives, Newton method, application to shape optimization. INRIA-report No. 2555 (1995)

[32] M. Pierre and J.-R. Roche Computation of free surfaces in the electromagnetic shaping of liquid metals by optimization algorithms. Eur. J. Mech, B/Fluids, 10: 489-500, 1991.

[33] O. Pironneau. Optimal shape design for elliptic systems. Springer, New York, 1983.

[34] J.-R. Roche and J. Sokolowski Numerical methods for shape identification problems. Control Cybern. 25:867-894, 1996.

[35] J. Simon. Differentiation with respect to the domain in boundary value problems. Numer. Funct. Anal. Optimization 2:649-687, 1980.

[36] J. Sokolowski and J.-P. Zolesio. Introduction to Shape Optimization. Springer, Berlin, 1992.

[37] T. Tiihonen. Shape optimization and trial methods for free-boundary problems. RAIRO Model. Math. Anal. Numér., 31(7):805-825, 1997.

[38] J. Wloka. Partial Differential Equations. Cambridge University Press, Cambridge, 1987. 